\title{
TU/e EmonONEN

\section{The permittivity of a plasma at cyclotron resonance in large amplitude e.m. fields}

\section{Citation for published version (APA):}

Schram, D. C. (1970). The permittivity of a plasma at cyclotron resonance in large amplitude e.m. fields. Physica, 46(1), 97-118. https://doi.org/10.1016/0031-8914(70)90120-5

DOI:

10.1016/0031-8914(70)90120-5

Document status and date:

Published: 01/01/1970

\section{Document Version:}

Publisher's PDF, also known as Version of Record (includes final page, issue and volume numbers)

\section{Please check the document version of this publication:}

- A submitted manuscript is the version of the article upon submission and before peer-review. There can be important differences between the submitted version and the official published version of record. People interested in the research are advised to contact the author for the final version of the publication, or visit the $\mathrm{DOI}$ to the publisher's website.

- The final author version and the galley proof are versions of the publication after peer review.

- The final published version features the final layout of the paper including the volume, issue and page numbers.

Link to publication

\section{General rights}

Copyright and moral rights for the publications made accessible in the public portal are retained by the authors and/or other copyright owners and it is a condition of accessing publications that users recognise and abide by the legal requirements associated with these rights.

- Users may download and print one copy of any publication from the public portal for the purpose of private study or research.

- You may not further distribute the material or use it for any profit-making activity or commercial gain

- You may freely distribute the URL identifying the publication in the public portal.

If the publication is distributed under the terms of Article 25fa of the Dutch Copyright Act, indicated by the "Taverne" license above, please follow below link for the End User Agreement:

www.tue.nl/taverne

Take down policy

If you believe that this document breaches copyright please contact us at:

openaccess@tue.nl

providing details and we will investigate your claim. 


\section{THE PERMITTIVITY OF A PLASMA AT CYCLOTRON RESONANCE IN LARGE AMPLITUDE E.M. FIELDS \\ D. C. SCHRAMI \\ Association Euratom-FOM, FOM-Instituut voor Plasma-Fysica, Rijnhuizen, Jutphaas, Nederland}

Received 5 June 1969 .

\section{Synopsis}

The permittivity of a collisionless plasma as a function of field parameters is measured in standing and in travelling waves. In both experiments the permittivity remains finite at cyclotron resonance; the resonance is broadened and shifted towards higher values of the magnetic field strength. The results are in quantitative agreement with theoretical predictions which follow from a cold-plasma, large-signal analysis. The loss tangent of the plasma is measured. The loss is found to be due to the escape of accelerated particles.

1. Introduction. The relative value of the permittivity of a cold collisionless plasma in a spatially uniform right-handed circularly polarized TEMwave with $k$ parallel to a uniform axial magnetic field, $\boldsymbol{B}_{0}$, is ${ }^{1}$ ):

$$
\varepsilon=1-\frac{\omega_{\mathrm{p}}^{2}\left(\omega-k v_{z}\right)}{\left(\omega-k v_{z}-\Omega\right) \omega^{2}} .
$$

Here, $\omega_{\mathrm{p}}$ is the angular plasma frequency, $\Omega$ is the cyclotron frequency, $\omega$ is the applied frequency, $k$ is the wave number, and $v_{z}$ is the axial velocity of the electrons. The relativistic increase of the mass and the axially directed h.f. Lorentz force will cause $\Omega(t)$ and $v_{z}$ to be time-dependent quantities in eq. $(1)^{2,3}$. Consequently, the velocity and the energy of the electrons oscillate in time with an oscillation time $\tau_{\text {os }}$, cf. ref. 4 , fig. 2 . These effects are important in a region around cyclotron resonance; the width of this region depends on the electric field strength of the wave.

Throughout the paper we shall assume that a cold-plasma, large-signal analysis applies; the thermal energy must be small compared to the energy attained after acceleration in the fields. Also the axial drift velocity, $v_{\mathrm{zo}}$, which is retained in the treatment of ref. 4 is here assumed to be zero.

The assumption of zero thermal energy is realistic in our experimental situations, where the charged particles are produced by volume ionization 
and secondary emission in the h.f. field. The collisions are rare and occur most likely near the minimum of the energy oscillation, since ionization cross sections show a maximum around a few $10 \mathrm{eV}$. Also, the starting energy of secondary electrons is small. Thus, the majority of the electrons have small initial velocities.

The dielectric behaviour of a plasma in a travelling wave is described in ref. 4. The homogeneous electric field approximation of ref. 5, which in this study will be derived from ref. 4, can be applied to a standing wave. In both cases the permittivity is calculated by the integration over velocity space of that velocity component, which is out of phase with the electric field of the wave. Similarly, the loss tangent can be obtained by the integration of the in-phase velocity component. In the theoretical model of ref. 4 the loss tangent is zero, since the plasma is assumed to be collisionless and of infinite dimensions. However, in a tenuous, finite plasma, particles will escape axially from the plasma volume under the action of the radiation pressure. Taking into account the fact that the energy of the escaping electrons is removed from the plasma volume, the loss tangent of the plasma can be calculated. Throughout our analysis the ions are assumed to form an immobile neutralizing background.

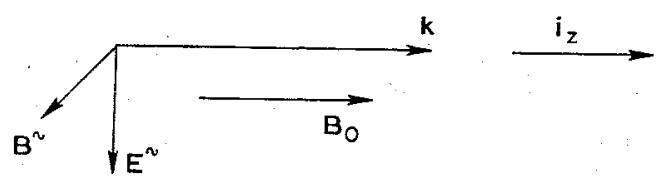

Fig. 1. Fields in a travelling wave.

1.1. Travelling wave. In the case of a travelling wave, $c f$. fig. 1 , the characteristic quantities of the interaction problem can be presented in a simple form by the use of three normalized parameters (cf. eq. (11) of ref. 4 with $v_{\mathrm{zo}} / v_{\mathrm{p}}=0$ ):

a density parameter.

$$
\alpha \equiv \omega_{\mathrm{p}}^{2} / \omega^{2}
$$

a parameter of the static magnetic field $\beta \equiv b-1$, with $b \equiv e B_{0} / m \omega=\Omega / \omega$;

and a parameter of the h.f. electric field $G \equiv g^{2}\left(1-n^{2}\right)$, with $g \equiv e \hat{E} / m \omega c$.

The total energy, $\mathscr{E}_{\text {totaj, }}$ normalized to $m c^{2}$ is:

$$
\gamma=\frac{\mathscr{E}_{\text {total }}}{m c^{2}}=\frac{m(t)}{m}
$$

where $m(t)$ is the apparent mass and $m$ is the rest mass, $\hat{E}$ is the amplitude of the electric field strength, and $n \equiv c / v_{\mathrm{p}}$ is the refractive index of the 
total system. Furthermore, a critical value of the parameter $\beta$ is:

$$
\beta_{\mathrm{c}} \equiv \frac{3}{2} \sqrt[3]{2 G}
$$

which is a measure of the resonance width.

It is shown in ref. 4 that for zero initial transverse velocity and for $|G| \ll 1$ the amplitude of the energy oscillation, the oscillation time $\left(t_{\mathrm{os}}\right)$, and the oscillation length $\left(z_{\mathrm{os}}\right)$ can be expressed as functions of $\beta / \beta_{\mathrm{c}}$. These functions, $\delta_{\text {extr }}^{\prime}, i_{0}, i_{1}$, are shown in figs. 3 and 4 of ref. 4 .

The amplitude is:

$$
\gamma-1=\frac{1}{1-n^{2}} \delta_{\text {extr }}^{\prime} G^{f},
$$

the normalized oscillation time is:

$$
\tau_{\mathrm{os}}=\omega t_{\mathrm{os}}=2\left|\frac{i_{1}}{1-n^{2}}+i_{0} G^{-\frac{1}{3}}\right|
$$

and the normalized oscillation length is:

$$
k z_{0 S}=2\left|\frac{n^{2}}{1-n^{2}}\right| i_{1}
$$

The permittivity of the plasma is expressed in the functions $i_{1} / i_{0}$ and $i_{2} / i_{0}$ which are shown in fig. 7 of ref. 4:

$$
1-\varepsilon=\alpha \frac{G^{-\frac{2}{3}} i_{2} / i_{0}}{G^{-\frac{1}{3}}+\frac{1}{1-n^{2}} \cdot \frac{i_{1}}{i_{0}}} .
$$

The loss factor is obtained from ref. 4, by introducing in eq. (26) a typical escape time $\tau_{\text {es }} \equiv \omega t_{\text {es }}$ as the upper limit of the integral:

$$
\operatorname{tg} \delta=\frac{i_{\eta} \hat{E}}{\omega \varepsilon_{0} \hat{E}^{2}}=\alpha \frac{\int_{0}^{\tau_{\mathrm{es}}} \frac{1}{g} \frac{v_{\eta}}{c} \mathrm{~d} \tau}{\tau_{\mathrm{es}}}=\alpha \frac{\delta_{\text {extr }}^{\prime} G^{-\frac{3}{3}}}{2 \tau_{\mathrm{es}}} .
$$

Here it is assumed that the average energy carried off by añ escaping electron is equal to half the maximum attainable energy. If the time of escape, $\tau_{\mathrm{es}}$, is $p$ times the characteristic time defined as $\frac{1}{2} \tau_{\mathrm{os}}$ :

$$
\tau_{\mathrm{es}} \equiv \frac{p}{2} \tau_{\mathrm{os}}
$$


we find:

$$
\operatorname{tg} \delta=\alpha \frac{\frac{\delta_{\text {extr }}^{\prime}}{2 p i_{0}} G^{-\frac{2}{3}}}{G^{-\frac{1}{3}}+\frac{1}{1-n^{2}} \cdot \frac{i_{1}}{i_{0}}} .
$$

Eq. (10) is a transcendental equation. It is solved in ref. 4 under the assumption that $n=\sqrt{ } \varepsilon_{\mathrm{r}}$. This implies that the interaction between the plasma and the wave takes place in a wave structure which, without a plasma, has $n_{0}=1$. With definition (4), eq. (9) can be written as a quadratic equation in $(1-\varepsilon)^{\frac{2}{3}}$. The solution is:

$$
1-\varepsilon=g\left[-\frac{i_{1}}{2 i_{0}}+\sqrt{\left(\frac{\overline{i_{1}}}{2 i_{0}}\right)^{2}+g^{-2} \alpha \frac{i_{2}}{i_{0}}}\right]^{\frac{3}{2}} .
$$

If $g$ and $\alpha$ are known the relationship between $(1-\varepsilon)$ and $\beta$ can be found by the following procedure: take a value of $\beta / \beta_{\mathrm{c}}$, calculate $1-\varepsilon$ and $\beta_{\mathrm{c}}$, and find $\beta$. For some values of the parameters the dependence of $(\varepsilon-1)$ is shown as a function of $\beta$ in fig. 8 of ref. 4 . The resonance is broadened symmetrically around $\beta=0$ and in an experimental situation a hysteresis must occur if the magnetic field is increased or decreased.

In the case that the interaction takes place in a slow or in a fast wave structure $\left(n_{0} \neq 1\right)$ the situation is more complex. Assume that the index of refraction, $n$, of the total system is a known function of the index of refraction, $n_{0}$, of the structure without plasma and of the index of refraction of the plasma, $\sqrt{ } \varepsilon$. Then an equation equivalent to that leading to the solution (14) can be found. In general this equation will be of higher order and it is difficult to obtain its roots. Once these roots have been obtained the functional dependence of $\varepsilon-1$ on $\beta$ can be calculated for each set of parameters $\alpha$ and $g$, following the procedure sketched above. From eq. (10) the value, $\beta_{0}$, of the magnetic field parameter where $\varepsilon=1$ can be obtained immediately. As in this case $1-n^{2}=1-n_{0}^{2} \neq 0$, the function $i_{2} / i_{0}$ must be zero which occurs for $\beta / \beta_{\mathrm{c}} \simeq 0.7$. Therefore (ct. eqs. (4) and (6)),

$$
\beta_{0} \simeq 1.34 \sqrt[3]{g^{2}\left(1-n_{0}^{2}\right)}
$$

As a result, in a fast wave the resonance is shifted towards higher values of the magnetic field and in a slow wave, towards lower values of $\beta$. A hysteresis may still occur for densities at which $|\varepsilon-1|$ is larger than $\left|n_{0}^{2}-1\right|$ close to the resonance.

For lower values of the density the permittivity of the plasma remains close to 1:

$$
|1-\varepsilon| \ll\left|1-n_{0}^{2}\right|,
$$


and

$$
G \simeq g^{2}\left(1-n_{0}^{2}\right)
$$

In this case the permittivity is (with $G \ll 1$ ):

$$
1-\varepsilon \cong \alpha G^{-\frac{1}{3}} i_{2} / i_{0}
$$

while the loss tangent is:

$$
\operatorname{tg} \delta \simeq \alpha G^{-\frac{1}{\delta}} \frac{\delta_{\text {extr }}^{\prime}}{2 p i_{0}}
$$

The value of $p$ for axial escape can be estimated as the ratio between the total interaction length, $L$, and the oscillation length, $z_{\mathrm{OS}}$ :

$$
p=\frac{\tau_{\mathrm{es}}}{\frac{1}{2} \tau_{\mathrm{os}}} \simeq \frac{k L}{\frac{1}{2} k z_{\mathrm{os}}}=\frac{k L\left(1-n_{0}^{2}\right)}{n_{0}^{2} i_{1}} .
$$

1.2. Standing wave. The interaction in a standing wave (fig. 2) has been treated $\left.{ }^{5}\right)$ in the approximation of a homogeneous $\hat{E}$-field $\left(n_{0}=0\right)$ where the magnetic field of the wave is neglected. In a microwave cavity this description is almost exact for the antinode of the electric field of the standing wave. Outside the antinode there still exists an axial acceleration,

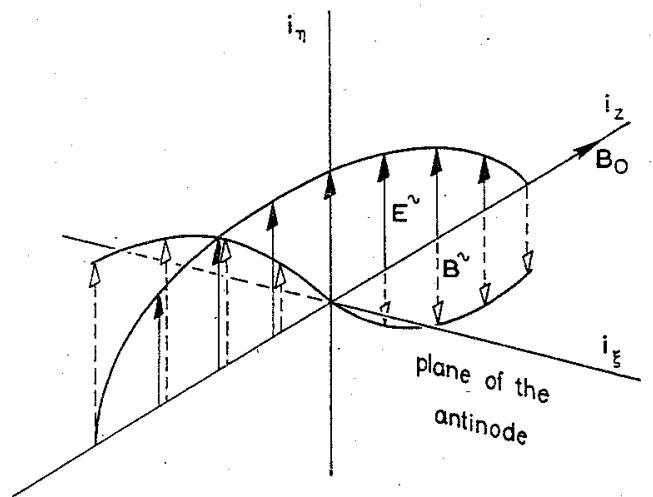

Fig. 2. Fields in a standing wave.

but on the base of qualitative arguments 5 ) we conclude that its effect can be neglected for the evaluation of the permittivity. In the homogeneous $\hat{E}$-field description the electric fieid parameter is equal to $G=g^{2}$, where $g=e \hat{E} / m \omega c$ and $\hat{E}$ is the amplitude of the electric field in the antinode of the standing wave. Again, $\beta_{\mathrm{c}} \simeq \frac{3}{2} \sqrt[3]{2 G}$. The amplitude of the energy oscillation, the oscillation time, the permittivity, and the loss tangent can be obtained from eqs. (7), (8), (10) and (11) with $n^{2}=0$. For the permittivity and the loss tangent we obtain eqs. (18) and (19) respectively, while here $G=g^{2}$. 
The function $i_{2} / i_{0}$ is sketched in fig. 7 and $\delta_{\text {extr }}^{\prime} / 2 p i_{0}$ is shown in fig. 8 for various values of $p$. The resonance is broadened and shifted towards higher values of the magnetic field strength.

Again, for the case of axial escape an estimate of the ratio $p$ between $\tau_{\mathrm{es}}$ and $\tau_{\mathrm{os}} / 2$ may be obtained by a rough integration of the axial equation of motion, eq. (9) of ref. 5. It follows that the increase in axial velocity during an oscillation time is:

$$
\Delta \frac{v_{z}}{c} \simeq i_{2} g^{\frac{2}{3}}
$$

Taking this quantity as the average velocity during the time that the particle travels through the cavity of length $L$ we find for $p$ :

$$
p=\frac{\tau_{\mathrm{es}}}{\frac{1}{2} \tau_{\mathrm{os}}} \approx \frac{l k_{n m} L}{\left\langle v_{z} / c\right\rangle i_{0} g^{-\frac{2}{3}}} \cong \frac{l k_{n m} L}{i_{2} i_{0}}
$$

where $k_{n m}$ is the wave number of the cavity mode: TE $E_{m m l}$. It must be emphasized that eq. (21) gives only an indication about the order of magnitude of $p$ and is only applicable for a situation where the electrons are accelerated out of the maximum field region $\left.\left(\beta / \beta_{\mathrm{c}}<0.7\right)^{6}\right)$.

2. Standing-rave experiment. The first attempt to verify the nonlinear behaviour of the permittivity of a plasma at cyclotron resonance has been done in a circularly polarized $\mathrm{TE}_{113}$ mode in a cylindrical cavity. From preliminary experiments ${ }^{7}$ ) in a linearly polarized standing wave it turned out to be necessary to use a right-handed circular polarization. In the case of linear polarization it was difficult or even impossible to distinguish the heavily damped right-handed* mode from the left-handed mode, especially at $\beta=\beta_{0}$ where the resonance frequencies are close.

A circular polarization may be accomplished in a large part of the cavity by the use of $\mathrm{TE}_{11 l}$ modes, cf. fig. 3a. By making both the input coupling and the output coupling optimal for the right-handed mode (fig. 4a) we have obtained a discrimination of $40 \mathrm{~dB}$ against the left-handed mode. In order to arrive at this result it was necessary to tune the cavity in such a way that the resonance frequencies of the four linear polarizations I, II, III, and IV (cf. fig. $4 \mathrm{~b}$ ) were equal. To this end a tuning element has been inserted into the cavity.

In order to increase the time of axiai escape of the particles a triple overmode in the $z$ direction is used: $T E_{113}$ mode.

* These modes are labelled left-handed and right-handed modes though the polarization is not purely circular throughout the volume; e.g. at the wall the polarization is linear. 

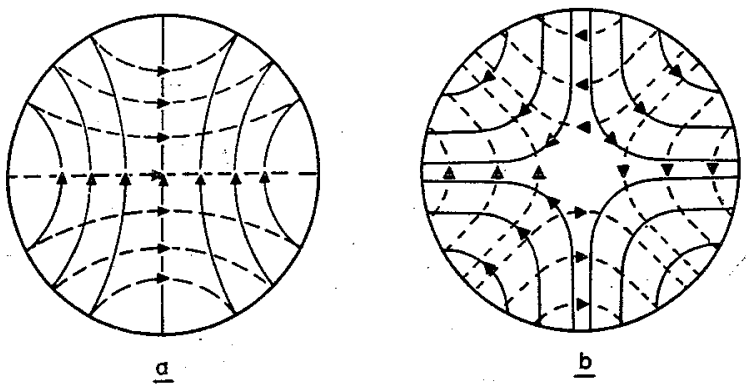

Fig. 3. Electric field patterns of two orthogonal, linearly polarized modes;

a) $\mathrm{TE}_{11 l}$ modes; b) $\mathrm{TE}_{21 l}$ modes.

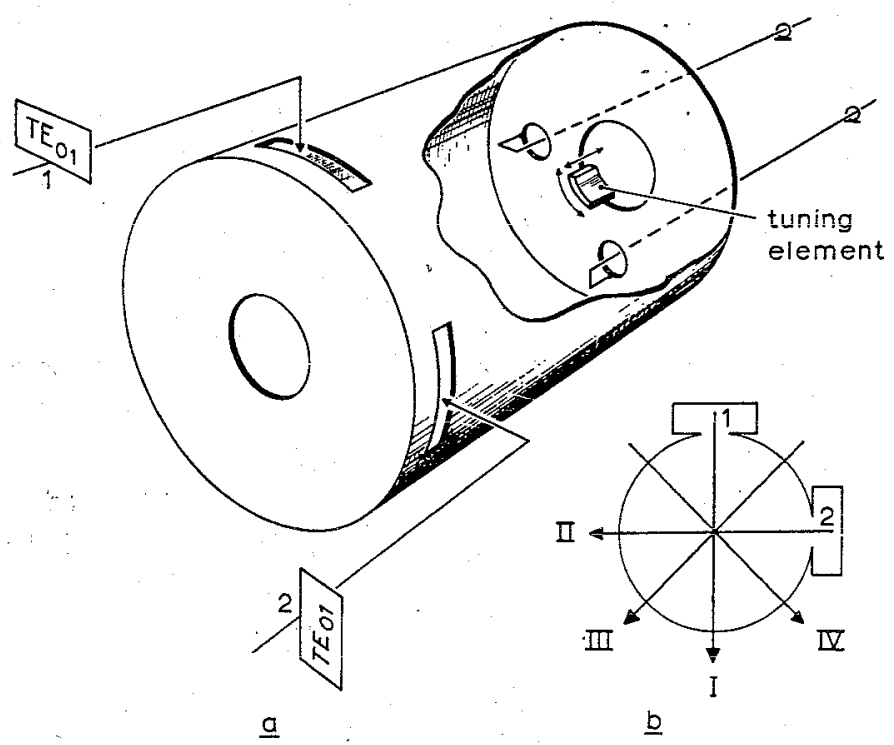

Fig. 4a. Sketch of input coupling and output coupling of the cavity.

Fig. 4b. Linear polarizations in four directions; I, II signal in wave guides 1), 2), respectively; III, IV signal in both wave guides 1) and 2) and with a phase difference of $0^{\circ}$ and $180^{\circ}$, respectively.

2.1. Experimental arrangement. The experimental arrangement is shown in fig. 5. A frequency-stabilized (stabilization factor 10-5) backwardwave oscillator (1) is used as the h.f. source. The maximum value of the power, $P$, delivered by the source is $200 \bar{W} \bar{C} \bar{W}$. The spiitting of the signal and a $90^{\circ}$ phase shift between the two branches is obtained by the use of a $3 \mathrm{~dB}$-quadrature hybrid (4). The power, $P_{\text {refl }}$, reflected from the cavity (3) goes mainly through the fourth port of the hybrid. The dimensions of the cavity are: length $I=0.153 \mathrm{~m}$ and diameter $2 R=0.077 \mathrm{~m}$; the resonance frequency of the $\mathrm{TE}_{113}$ mode is $f=3.6934 \mathrm{GHz}$, and the unloaded quality 


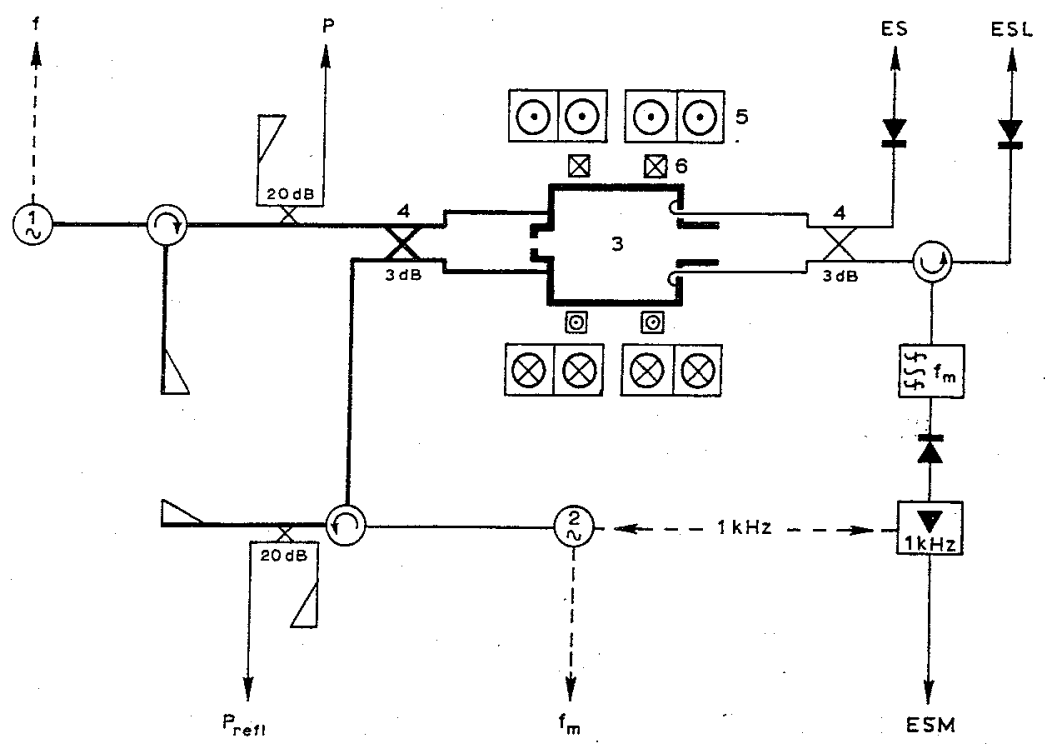

Fig. 5. Schematic diagram of the standing wave experiment; see text.

factor is $Q_{0}=15000$. The cavity can be pumped down to pressures of $10^{-7}$ torr; working pressures are up to $10^{-4}$ torr. The frequency shift, $\Delta f$, caused by the permittivity of the plasma is measured with the help of a transfer oscillator and a counter. By the use of a second $3 \mathrm{~dB}$ quadrature hybrid at the outputs of the cavity, the resonant right-handed signal can be measured at "ES" whereas the residual left-handed signal is measured at "ESL". The latter is measured for the distinction of the resonance of the right-handed from that of the left-handed mode.

A $1 \mathrm{kHz}$, A.M.-modulated klystron source (2) is tuned to the $\mathrm{TE}_{211}$ mode (fig. 3b) of the cavity. The resonant frequency of this so called "measuring mode" is $f_{m}=3.906 \mathrm{GHz}$, which is chosen relatively far from cyclotron resonance. From the frequency shifts, $\Delta t_{\mathrm{mlh}}$ and $\Delta t_{\mathrm{mrh}}$, of the left- and righthanded $T E_{211}$ modes the density of the plasma is obtained. The resonance of these modes is monitored at "ESM".

The static magnetic field, $B_{0}$, is produced by a current stabilized to $1 / 10^{5}$ which flows through four coils (5) and two compensation coils (6). On the axis the inhomogeneity of the magnetic field is smaller than $0.5 \%$ over the length of the cavity.

In the course of the experiment the magnetic field has been varied and subsequently the frequency of the backward wave oscillator was tuned to the shifted resonant frequency of the right-handed $\mathrm{TE}_{113}$ mode.

The measured quantities are summarized in table I: 
THE PERMITTIVITY OF A PLASMA AT CYCLOTRON RESONANCE 105

TABLE I

\begin{tabular}{|c|c|c|}
\hline quantity & measured & yields \\
\hline $\begin{array}{l}B_{0} \\
f\end{array}$ & $\begin{array}{l}\text { current through coils } \\
\text { frequency shift, } \Delta f \text {, of the } \mathrm{rh} \mathrm{TE}_{113} \text { mode }\end{array}$ & $\begin{array}{l}\text { magnetic field } \\
\text { parameter } \beta \\
\text { permittivity } \varepsilon\end{array}$ \\
\hline$f_{\mathbf{m}}$ & $\begin{array}{l}\text { frequency shifts } \Delta f_{\mathrm{min}}, \Delta f_{\mathrm{mrh}} \text { of the rh } \\
\quad \text { and lh } \mathrm{TE}_{211} \text { modes }\end{array}$ & $\begin{array}{l}\text { density parameter } \alpha \text {, } \\
\text { information about the } \\
\text { density profile }\end{array}$ \\
\hline $\begin{array}{l}P \\
P_{\text {rep }}\end{array}$ & $\begin{array}{l}\text { input power } \\
\text { reflected power }\end{array}$ & electric field parameter $G$ \\
\hline$E S$ & $\begin{array}{l}\text { square of the amplitude of the electric } \\
\text { field of the } T E_{113} \text { mode }\end{array}$ & unloaded quality factor $Q_{0}$ \\
\hline
\end{tabular}

2.2. Field parameter $G$ and loss tangent: The field parameter $G$ for a right-handed polarization with amplitude $\hat{E}$

$$
G \equiv\left(\frac{e \hat{E}}{m \omega c}\right)^{2}
$$

follows from the stored energy ${ }^{8}$ ):

$$
\int_{v} W \mathrm{~d} V=0.238 V_{\text {cavity }} \varepsilon_{0} \hat{E}^{2}=\frac{P_{\text {in }} Q_{0}}{\omega},
$$

where $P_{\text {in }}=P-P_{\text {repl }}$.

Without a plasma the input coupling is critical, at the resonance frequency the reflected power is zero, and the electric field parameter, $G[0]$, is:

$$
G[0]=0.21 \times 10^{-6} P_{\mathrm{in}}[0] .
$$

In the presence of the plasma the change of the electric field parameter $G[n]$ is measured at "ES" and:

$$
G[n]=\frac{E S[n]}{E S[0]} \cdot G[0] .
$$

The unloaded quality factor of the cavity can be calculated as follows:

$$
Q_{0}[n]=Q_{0}[0] \frac{E S[n] \cdot P_{\text {in }}[0]}{E S[0] \cdot P_{\text {in }}[n]}
$$

If $P_{\text {repl }}[n] / P[n]>0.9$ an alternative, approximate, formula is used:

$$
Q_{0}[n] \simeq \frac{1}{2} Q_{0}[0] \sqrt{\frac{E S[n]}{E S[0]}} .
$$

The loss tangent of the plasma is:

$$
\operatorname{tg} \delta=1 / Q_{0}[n]-1 / Q_{0}[0] \text {. }
$$


2.3. Shift of the resonance frequencies of $\mathrm{TE}_{n m l}$ modes. Both the $\mathrm{TE}_{113}$ and $\mathrm{TE}_{211}$ modes are degenerate: without a plasma two orthogonal modes (see fig. 3) have identical frequencies. In the presence of a plasma in an axial static magnetic field this degeneracy is removed and two modes with different resonant frequencies, $f_{\mathrm{rh}}$ and $f_{\mathrm{ln}}$, result. The resonant frequencies of the right-handed ( $\mathrm{rh}$ ) and left-handed (lh) modes follow from 8,9 ):

$$
\begin{aligned}
& -2\left(\frac{\Delta t}{f}\right)_{\mathrm{rh}}= \\
& \quad=\frac{\iiint \omega_{\mathrm{p}}^{2}\left(E_{r 1}^{2}+E_{\varphi 1}^{2}\right) \mathrm{d} V \pm(\Omega / \omega) \iiint \omega^{2}\left(E_{r 1} E_{\varphi 2}-E_{r 2} E_{\varphi 1}\right) \mathrm{d} V}{(\Omega+\omega)(\Omega-\omega) \iiint\left(E_{r 1}^{2}+E_{\varphi 1}^{2}\right) \mathrm{d} V},
\end{aligned}
$$

where the minus sign applies to $\Delta f_{\mathrm{ln}}$ and the plus sign to $\Delta f_{\mathrm{rh}}$. The fields of the two orthogonal linearly polarized $\mathrm{TE}_{n m l}$ modes are

$$
\begin{aligned}
& E_{r 1,2}=E_{0} \frac{n}{k_{n m^{\gamma}}} J_{n}\left(k_{n m} \gamma\right)\left\{\begin{array}{l}
\sin n \varphi \\
\cos n \varphi
\end{array}\right\} \cos \frac{l k z}{2} ; \\
& E_{\varphi 1,2}=E_{0} J_{n}^{\prime}\left(k_{n m} \gamma\right)\left\{\begin{array}{l}
\cos n \varphi \\
\sin n \varphi
\end{array}\right\} \cos \frac{l k z}{2} .
\end{aligned}
$$

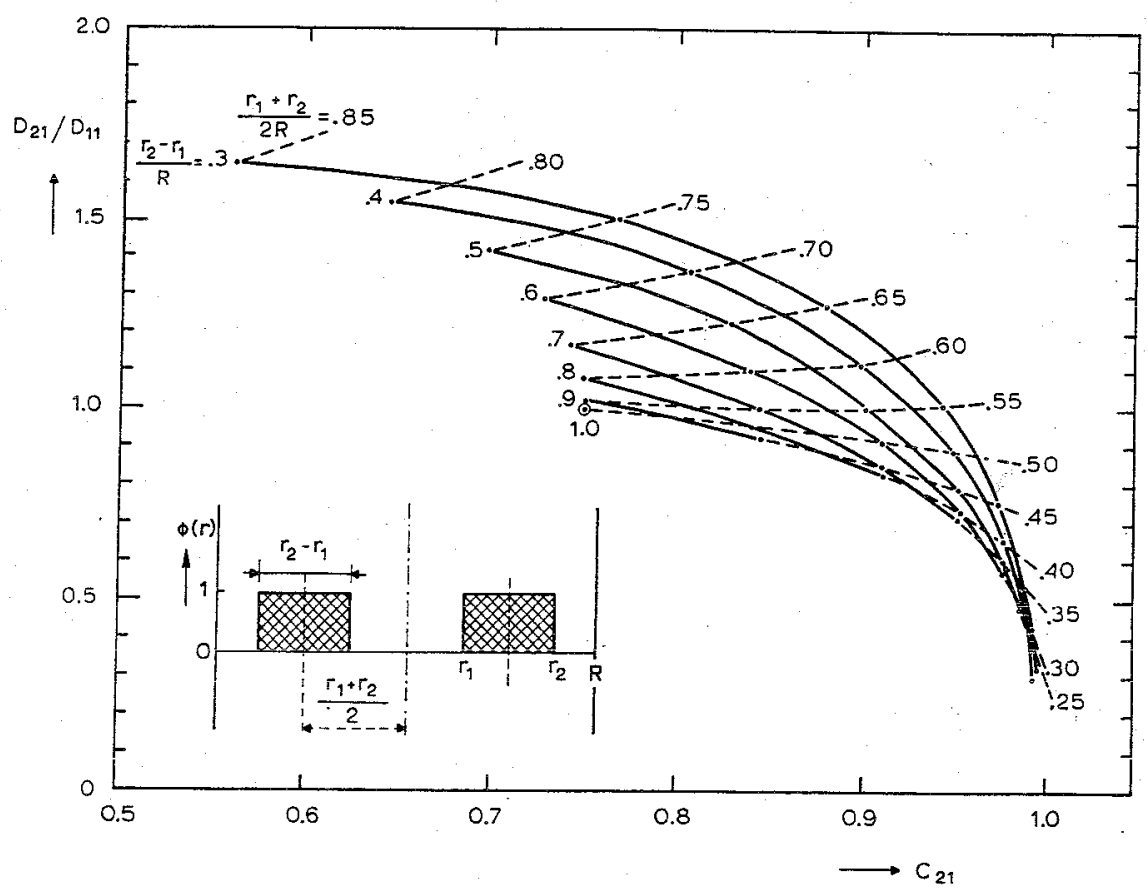

Fig. 6. Plot of $D_{21} / D_{11}$ as a function of $C_{21}$ for rectangular density profiles; $\left(\gamma_{2}-\gamma_{1}\right) / R$ is the width, and $\left(\gamma_{1}+\gamma_{2}\right) / 2 R$ is the average position of the profile. 
Firstly, we suppose that the density distribution is axially symmetric and that we may separate the radial and axial dependences of the density:

$$
\omega_{\mathrm{p}}^{2}(r, z)=\hat{\omega}_{\mathrm{p}}^{2} \cdot \Phi(r) \cdot \psi(z) .
$$

Furthermore, we assume that the density profile in the $r$ direction is rectangular (cf. insert in fig. 6): $\Phi(r)=1$ for $r_{1}<r<r_{2}$, and elsewhere $\Phi(r)=0$. In that case the resonant frequencies of the rh and $\mathrm{lh} \mathrm{TE}_{n m l}$ modes are given by:

$$
-2\left(\frac{\Delta f}{f}\right)_{\mathrm{lh}}=\frac{1 \pm \frac{\Omega}{\omega} C_{n m}}{(\Omega+\omega)(\Omega-\omega)} D_{n m} E_{l} \hat{\omega}_{\mathrm{p}}^{2}
$$

where:

$$
\begin{aligned}
C_{n m} & =\frac{\left(\frac{n}{2} J_{n}^{2}\left(k_{n m} \gamma\right)\right)_{r=r_{1}}^{r=r_{2}}}{\left(\frac{k_{n m}^{2} r^{2}}{2}\left(J_{n-1}^{2}-J_{n-2} J_{n}\right)-n J_{n}^{2}\right)_{r=r_{1}}^{r=r_{2}}} \\
D_{n m} & =\frac{\left(\frac{k_{n m^{\gamma^{2}}}^{2}}{2}\left(J_{n-1}^{2}-J_{n-2} J_{n}\right)-n J_{n}^{2}\right)_{r=r_{1}}^{r=r_{2}}}{\left(\frac{k_{n m}^{2} \gamma^{2}}{2}\left(J_{n-1}^{2}-J_{n-2} J_{n}\right)-n J_{n}^{2}\right)_{r=0}^{r=R}} \\
E_{l} & =\frac{\int \psi(z) \cos ^{2} \frac{1}{2} k l z \mathrm{~d} k z}{\int \cos ^{2} \frac{1}{2} k l z \mathrm{~d} k z} .
\end{aligned}
$$

Observe, that the terms $C$ and $D$ are only dependent on $n$ and $m$, and $\dot{E}$ only on $l$. In fig. 6 the ratio $D_{21} / D_{11}$ is plotted as a function of $C_{21}$ for hollow rectangular profiles; the special case $r_{1}=0$ yields of course a solid profile.

From measurements of the shifts $\Delta f_{\mathrm{mrh}}$ and $\Delta f_{\mathrm{mlh}}$ of the resonant frequencies of the rh- and lh-TE $E_{211}$ modes the quantities $C_{21}$ and $D_{21} E_{1} \hat{\omega}_{\mathrm{p}}^{2}$ are obtained:

$$
\begin{aligned}
D_{21} \hat{\alpha} E_{1} & =D_{21} E_{1} \frac{\hat{\omega}_{\mathrm{p}}^{2}}{\omega^{2}}= \\
= & \frac{-f_{\text {min }}\left(\beta_{\text {min }} \Delta f_{\min }+\beta_{\operatorname{mrh}} \Delta f_{\operatorname{mrh}}\right)\left(2+\beta_{\mathrm{m}}\right)}{f^{2}},
\end{aligned}
$$

where $\hat{\alpha} \equiv \hat{\omega}_{\mathrm{p}}^{2} / \omega^{2}$.

$$
C_{21}=\frac{\beta_{\mathrm{mrh}} \Delta f_{\mathrm{mrh}}-\beta_{\mathrm{mlh}} \Delta f_{\mathrm{mlh}}}{\left(\beta_{\mathrm{mrh}} \Delta f_{\mathrm{mrh}}+\beta_{\mathrm{mlh}} \Delta f_{\mathrm{mlh}}\right)\left(\beta_{\mathrm{m}}+1\right)} .
$$


Here:

$$
\beta_{\mathrm{mrh}}=\frac{\Omega}{2 \pi\left(f_{\mathrm{m}}+\Delta f_{\mathrm{mrh}}\right)}-1, \quad \beta_{\mathrm{mlh}}=\frac{\Omega}{2 \pi\left(f_{\mathrm{m}}+\Delta f_{\mathrm{mlh}}\right)}-1,
$$

and

$$
\beta_{\mathrm{m}}=\frac{\Omega}{2 \pi f_{\mathrm{m}}}-1
$$

The resonant frequency of the $T E_{113}$ mode is chosen close to the cyclotron frequency $-\beta_{\mathrm{c}}<\beta<2 \beta_{\mathrm{c}}$; the term $\omega /(\Omega-\omega)$ in the denominator of eq. (30) has to be replaced by its relativistic equivalent

$$
\frac{\varepsilon-1}{\alpha}=-\frac{i_{2}}{i_{0}} G^{-\frac{1}{3}}
$$

and it follows:

$$
-2 \frac{\Delta f}{f}=\frac{1+C_{11}(\beta+1)}{2+\beta} \frac{i_{2}}{i_{0}} G^{-\frac{1}{8}} \frac{D_{11} E_{3}}{D_{21} E_{1}}\left(D_{21} E_{1} \hat{\alpha}\right) .
$$

Here $\varepsilon$ is the permittivity of a plasma in a purely right-handed circular polarized wave.

The loss tangent should be equal to, cf. eq. (19):

$$
\operatorname{tg} \delta \cong \frac{\delta_{\text {extr }}^{\prime}}{2 p i_{0}} G^{-\frac{1}{3}} \frac{D_{11} E_{3}}{D_{21} E_{1}}\left(D_{21} E_{1} \hat{x}\right)
$$

2.4. Experimental data and conclusions. According to eqs. (18) and (34) the quantity $(\varepsilon-1) G^{\frac{z}{z}} / \alpha$, which is related to the permittivity, depends only on the normalized magnetic field parameter $\beta / \beta_{\mathrm{c}}$. Similarly, it follows from eq. (35) that the same applies to the quantity $(\operatorname{tg} \delta) G^{\frac{3}{3}} / \alpha$ which is related to the loss tangent. In figs. 7 and 8 the measured values of these reduced quantities are compared to the theoretical values of $i_{2} / i_{0}$ and $\delta_{\text {extr }}^{\prime} / 2 p i_{0}$, respectively. In fig. 8 curves for various fixed values of $p$, as well as the estimated

$$
p \simeq \frac{k_{n m} l L}{i_{2} i_{0}} \simeq \frac{10}{i_{2} i_{0}}
$$

(eq. 21) are plotted. Here, a homogeneous density distribution is assumed: $C_{11}=0.842, C_{21}=0.750, D_{11} / D_{21}=1$, and $E_{1} / E_{3}=1$. The presentation in normalized form of the experimental results permits to compare all experimental points to one normalized curve which is independent of the density and of the field strength of the wave. It should be noted that in the experiment both the density and the field strength vary over more than 


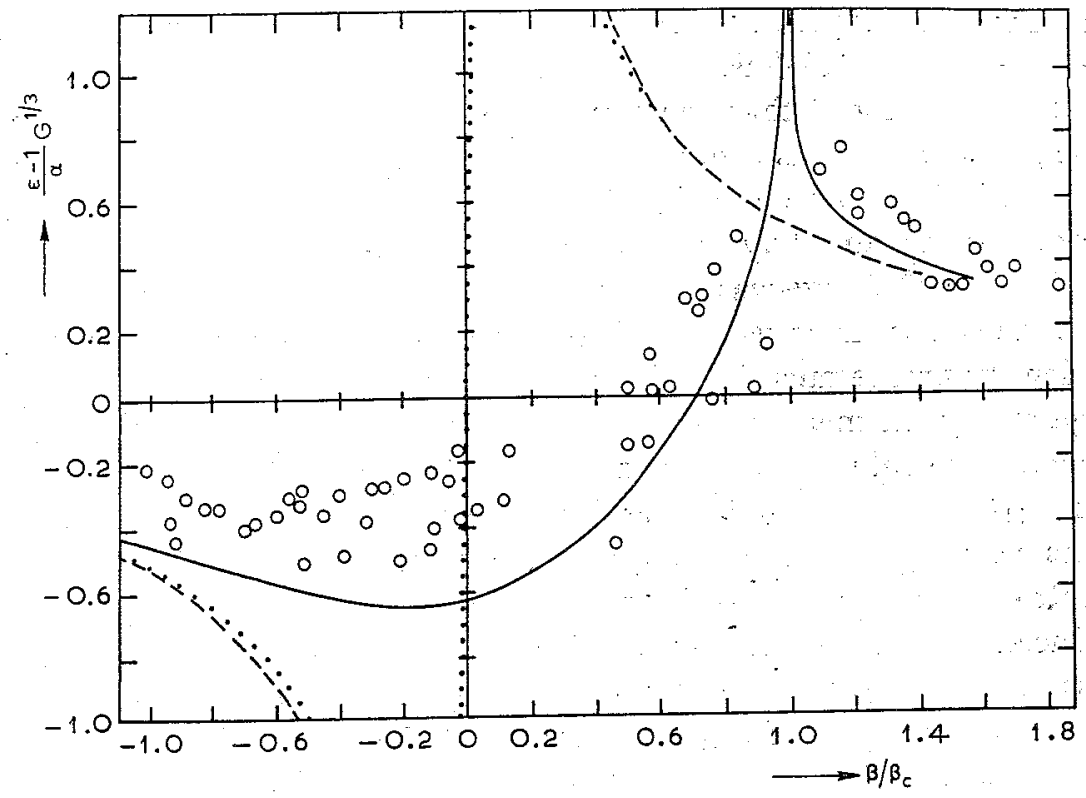

Fig. 7. Experimentally obtained values of $(\varepsilon-1) G^{\frac{1}{3}} / \alpha$ as a function of $\beta / \beta_{\mathrm{c}}$. The solid curve represents the theoretically predicted function $i_{2} / i_{0}$; the dashed curve is the nonrelativistic equivalent which follows from $\varepsilon-1=\alpha / \beta$; the dotted curve is the nonrelativistic curve corrected for the measured loss.

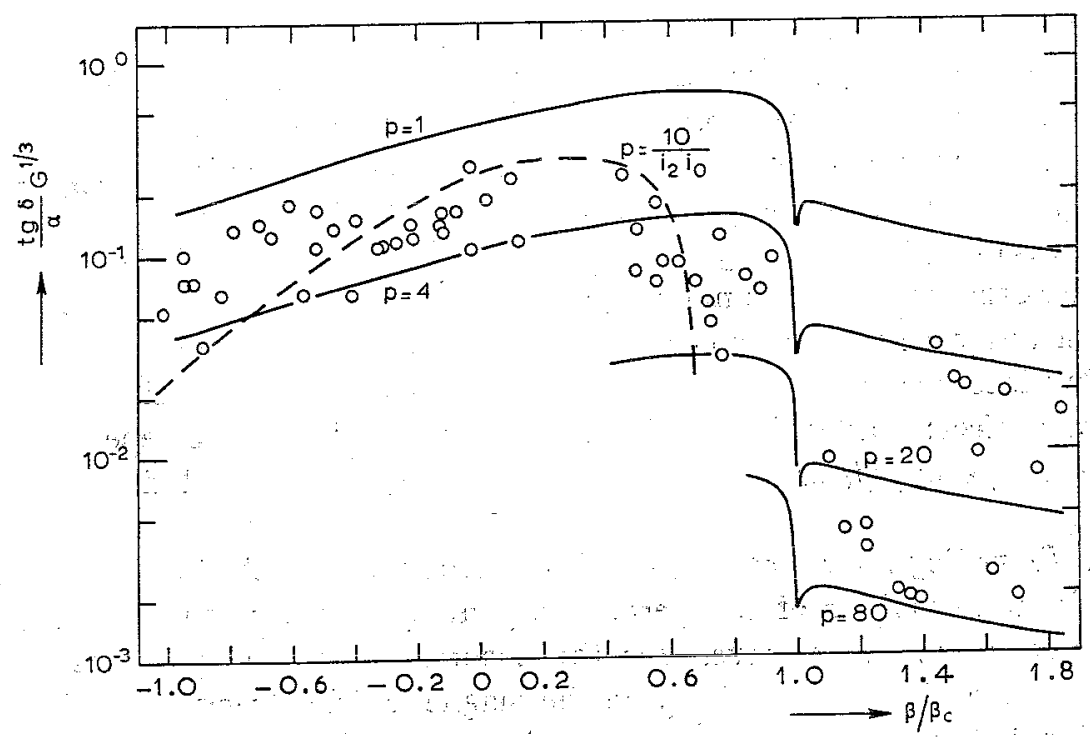

Fig. 8. Experimentally obtained values of $(\operatorname{tg} \delta / \alpha) G^{3}$ as a function of $\beta / \beta_{\mathrm{c}}$. The solid curves labelled $p=1,4,20,80$ give the predicted loss factors for fixed values of $p$. The dashed curve labelled $p=10 / i_{2} i_{0}$ shows the loss factor calculated for axial escape 
one order of magnitude $\left(\hat{E} \sim 3-40 \mathrm{kV} / \mathrm{m}, G \sim 5 \times 10^{-8}-8 \times 10^{-1}\right.$ $\left.n_{\mathrm{e}} \sim 10^{13}-2 \times 10^{14} / \mathrm{m}^{3}, \alpha \sim 5 \times 10^{-5}-10^{-3}\right)$.

It is seen in fig.. 7 that the experimental results confirm the theoretic: model, though there is a numerical discrepancy below cyclotron resonanc $\left(\beta<\beta_{\mathrm{c}}\right)$. This may be explained as follows. Below cyclotron resonance th electrons are produced by single-wall multipacting 8,10 ) as is confirmed by th fact that the discharge was formed even at pressures as low as $2 \times 10^{-7}$ torn Since the end walls have holes of 0.4 times the total diameter of the cavity the density profile must be hollow. The existence of such a hollow profile $i$ suggested by the measurement of $C_{21}$ of the $\mathrm{TE}_{211}$ mode, $c f$. eq. (32). Belor resonance the values of $C_{21}$ are between 0.7 and 0.8 in fig. 6 , and it follow that $D_{11} / D_{21} \simeq 1.0-1.5$. Therefore, the magnitude of the experimenta points below cyclotron resonance in fig. 7 must be increased by $25 \%$. Abov cyclotron resonance $\left(\beta>\beta_{c}\right)$ the situation is different: the electrons ar produced by volume ionization. The discharge could only be formed a: pressures higher than $10^{-5}$ torr. A convex profile exists, which is suggestec by the higher values of $C_{21}$, measured as 0.85 to 0.95 (cf. fig. 6). Now the correction is small.

From fig. 8 we may draw the conclusion that for $\beta / \beta_{\mathrm{c}}<1$ axial particle escape is indeed the dominant loss mechanism since the experimental findings indicate a low value of $p(\simeq 3)$ which is in the same order of magnitude as the value of $p \simeq 10 / i_{2} i_{0}$ for axial escape. Above resonance $\left(\beta / \beta_{\mathrm{c}}>1\right)$ the particles are confined in the maximum field regions, as is confirmed by the relatively small losses. Still, in our experiment the axial escape of a particle is possible, as seen by comparing the measured $p$-values with the numerical results of ref. 6. A small contribution of collisional damping cannot be excluded. In the presence of plasma the quality factor of the cavity is reduced to $Q_{0}[n] \sim 150-1000$.

The loading of the cavity will also damp the cyclotron resonance. In order to check that this effect is not responsible for the observed limitation of the permittivity we have calculated the dependence of the permittivity on $\beta / \beta_{\mathrm{c}}$ taking into account the measured loss tangent and disregarding the relativistic effect: dotted line in fig. 7. It is concluded that the dependence of $(\varepsilon-1) G^{\frac{1}{3}} / \alpha$ on $\beta / \beta_{\mathrm{c}}$ can only be explained by the nonlinear relativistic effect.

3. Travelling wave experiment. The second approach to measure the nonlinear behaviour of the permittivity at cyclotron resonance has been done in a travelling wave. For the occurrence of the hysteresis in the dependence of the permittivity on the magnetic field strength, predicted in section 1.1, it is favourable that the index of refraction, $n_{0}$, of the wave structure is equal to 1. We have chosen a TEM parallel-plate transmission line, in which the polarization can only be linear. The measured value of the permittivity, $\varepsilon_{\text {linear, }}$, is related to the permittivity, $\varepsilon$, in a right-handed 
circularly polarized wave as:

$$
\varepsilon-1=\left(1+\frac{\Omega}{\omega}\right)\left(\varepsilon_{\text {linear }}-1\right)=(2+\beta)\left(\varepsilon_{\text {linear }}-1\right) .
$$

Actually, the parallel-plate line used is found to be a fast wave structure with $n_{0}=0.87$. This may be caused by the generation of wave guide modes in the connections between the parallel-plate line and the coaxial input. The consequences hereof will be discussed in section 3.4.

3.1. Experimental arrangement. The experimental arrangement, sketched in fig. 9, consists of two microwave interferometers: a "main" interferometer to measure the phase shift of the signal at cyclotron resonance (frequency: $f$ ), and a "measuring" interferometer operated at a frequency, $f_{\mathrm{m}}$, for the measurement of the density. The h.f. sources are a backward-wave oscillator (1), $500 \mathrm{~W} \mathrm{CW}, f=2.84 \mathrm{GHz}$, stabilized to $1 / 10^{5}$, and a klystron oscillator (2), $50 \mathrm{~mW}$, amplitude-modulated with $1 \mathrm{kHz}$ for labelling purposes, $f_{\mathrm{m}}=2.55 \mathrm{GHz}$. The actual interaction chamber is a parallel-plate line (3) (see also fig. 10) of the following dimensions: length $L=0.22$, width

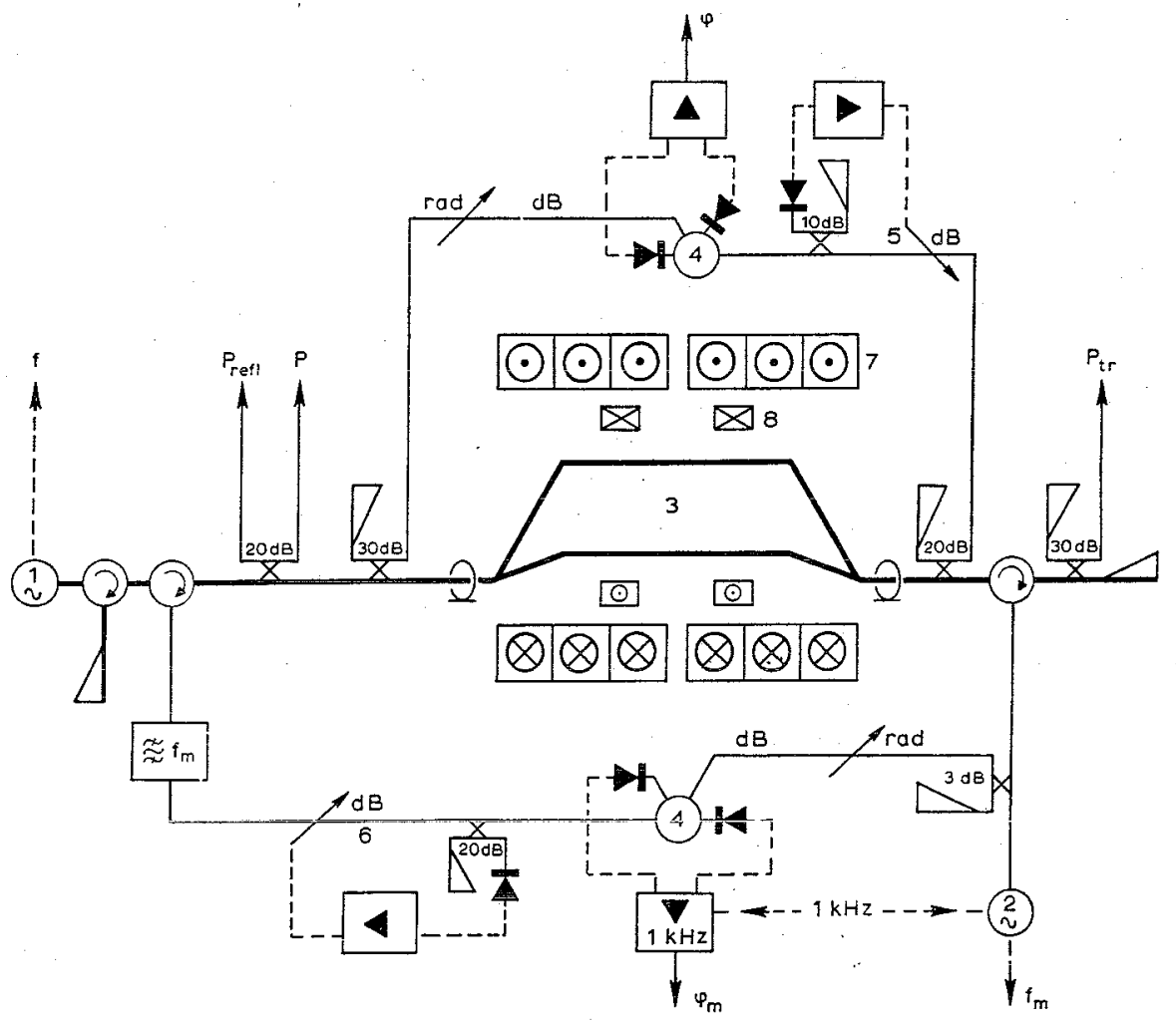

Fig. 9. Schematic diagram of the travelling wave experiment; see text. 


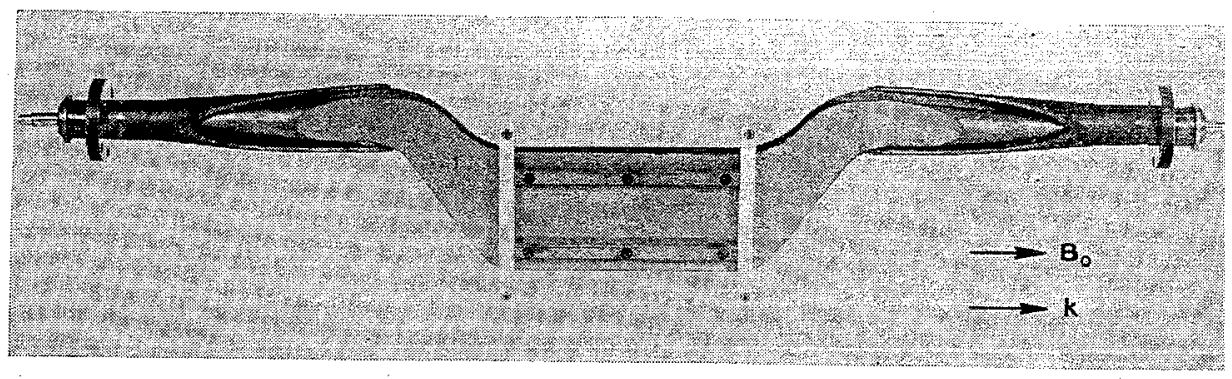

Fig. 10. Photo of the parallel-plate transmission line.

$0.08 \mathrm{~m}$, and spacing between the plates, $h=0.009 \mathrm{~m}$. The characteristic impedance, $Z_{0}$, for TEM-mode propagation is $50 \Omega$ which is equal to the characteristic impedance of the coaxial input and output. The voltage standing wave ratios at both ends of the parallel-plate line are smaller than 1.4 for both frequencies $f$ and $f_{\mathrm{m}}$. The parallel-plate line is enclosed in a vacuum vessel which can be pumped down to a pressure of $4 \times 10^{-7}$ torr.

The phase shifts, $\varphi$ and $\varphi_{\mathrm{m}}$; in the work paths of the two interferometers are measured at " $\varphi$ " and " $\varphi_{\mathrm{m}}$ ". The difference of the signals from the hybrid rings (4) is proportional to the phase shifts caused by the presence of plasma. This is valid under the conditions that the interferometers are nulled without a plasma, that the phase shift is not too large, and that the amplitudes of the signals at the hybrid ring remain constant. Therefore, in each interferometer the signal through the workpath is kept constant by means of a regulated attenuator (5), (6). The attenuator in the main interferometer is a PIN-diode attenuator (5) with a frequency response up to $10 \mathrm{kHz}$; that in the measuring interferometer is a motor-driven rotary-vane wave guide attenuator (6). The signals at " $\varphi$ " and " $\varphi_{\mathrm{m}}$ " have been calibrated with a known increase in phase in the nullpath.

The magnetic field is produced by a current, stabilized to $1 / 10^{5}$, which flows through six coils (7) and two compensation coils (8). The axial inhomogeneity is smaller than $0.8 \%$ over the total length of the parallel-plate

TABLE II

\begin{tabular}{lll}
\hline quantity & \multicolumn{1}{c}{ measured } & \multicolumn{1}{c}{ yields } \\
\hline$B_{0}$ & magnetic field strength \\
$f$ & frequency of the "main" wave $\}$ & magnetic fieid parameter $\beta$ \\
$P$ & input power $\}$ & electric field parameter $G$ \\
$P_{\text {repl }}$ & reflected power $\}$ & loss tangent \\
$P_{t r}$ & transmitted power & density parameter $\alpha$ \\
$\varphi_{\mathrm{m}}$ & phase shift of measuring wave & permittivity \\
$\varphi$ & phase shift of main wave & \\
\hline
\end{tabular}


line. The magnetic field parameter $\beta$ is varied by choosing different values for the magnetic field, while the frequency is kept constant.

The quantities measured are summarized in table II.

3.2. Field parameter $G$ and loss tangent. The electric field strength of the right-handed circularly polarized part of the wave is, with $P_{\text {in }}=P-P_{\text {refl }}$ :

$$
\hat{E} \equiv \hat{E}_{\mathbf{r h}}=\frac{1}{2} \hat{E}_{\text {linear }}=\frac{1}{2 h} \sqrt{2 P_{\text {in }} Z_{0}}=560 \sqrt{P_{\text {in }}} \quad V / m .
$$

In case $|1-\varepsilon| \ll\left|1-n_{0}^{2}\right|$, the field parameter $G$ is (cf. eq. (16)):

$$
G \simeq g^{2}\left(1-n_{0}^{2}\right)=\left(\frac{e \hat{E}}{m \omega c}\right)^{2}\left(1-n_{0}^{2}\right)
$$

The loss tangent is obtained by the measurement of the transmitted power, $P_{\text {tr }}$, in the plasma:

$$
\operatorname{tg} \delta=\frac{2.3}{k L} \operatorname{lol} \log \frac{P_{\text {tr }}}{P_{\text {in }}},
$$

where

$$
k L=\frac{2 \pi n_{0} L f}{c} .
$$

3.3. Phase shift in the parallel-plate line. For the dependence of the total index of refraction, $n$, on the index of refraction of the wave structure, $n_{0}$, and of the plasma, $\sqrt{ } \varepsilon$, we take:

$$
\varepsilon-n^{2}=1-n_{0}^{2}
$$

The permittivity of the plasma is related to the phase shift as follows (cf. eq. (40)):

$$
\varepsilon_{(\mathrm{m}) \text { linear }}-1=\left\{1-\frac{\Delta \varphi_{(\mathrm{m})} c}{2 \pi f_{(\mathrm{m})} n_{0} L}\right\}^{2} n_{0}^{2}-n_{0}^{2} \simeq \frac{\Delta \varphi_{(\mathrm{m})} n_{0} c}{\pi f_{(\mathrm{m})} L} .
$$

This applies both to the main interferometer and to the measuring $(\mathrm{m})$ interferometer. The frequency $f_{m}$ of the "measuring" wave is chosen far enough from cyclotron resonance so that the classical formula for $\varepsilon$ is valid:

$$
\varepsilon_{\text {minear }}-1=\frac{\omega_{\mathrm{p}}^{\bar{z}} / \omega_{\mathrm{m}}^{\bar{z}}}{\beta_{\mathrm{m}}\left(2+\beta_{\mathrm{m}}\right)},
$$

where

$$
\beta_{\mathrm{m}}=\frac{\Omega}{2 \pi f_{\mathrm{m}}}-1
$$



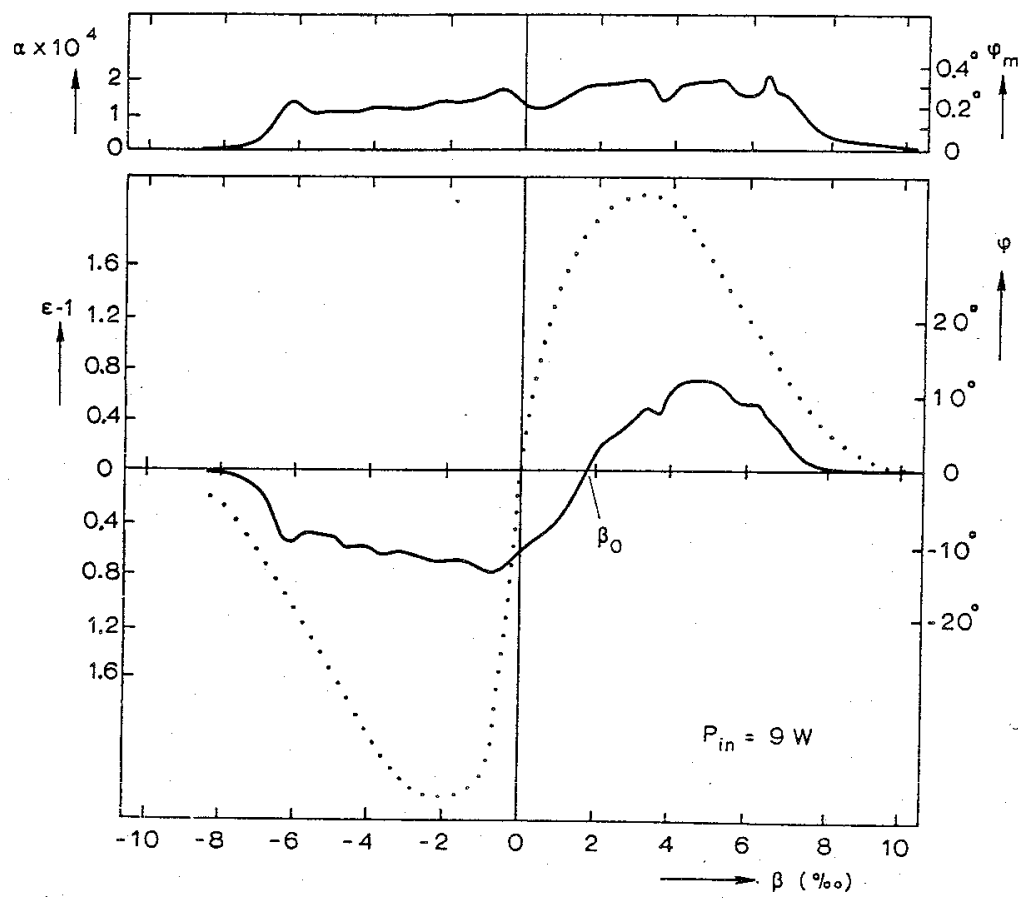

Fig. 11. Measured dependences of $(\varepsilon-1)$ and $\alpha$ on $\beta$ at low values of the density (full line); the loss dominated behaviour of $(\varepsilon-1)$ at high values of the density is sketched as a dotted line.

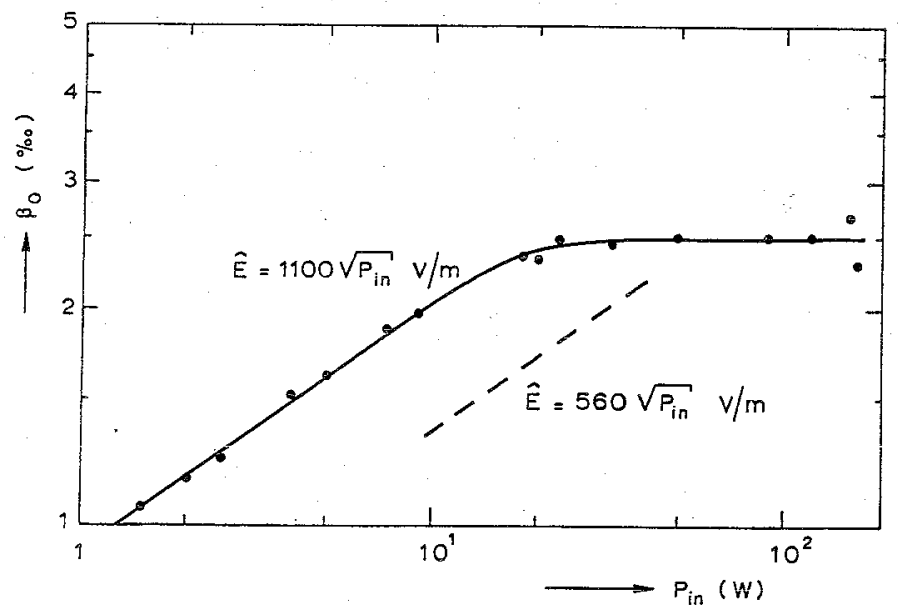

Fig. 12. $\beta_{0}$, the value of the magnetic field parameter where $\varepsilon-1=0$, as a function of $P_{\text {in. }}$. 
From eqs. (42) and (43) it follows for the density parameter $\alpha=\omega_{\mathrm{p}}^{2} / \omega^{2}$ :

$$
\alpha \cong \frac{\Delta \varphi_{\mathrm{m}} \cdot c n_{0} \beta_{\mathrm{m}}\left(2+\beta_{\mathrm{m}}\right)}{\pi L f_{\mathrm{m}}} \frac{f_{\mathrm{m}}^{2}}{f^{2}}
$$

The permittivity in a right-handed circularly polarized wave can be obtained from eqs. (36) and (41):

$$
\varepsilon-1=(2+\beta) \frac{\Delta \varphi \cdot c n_{0}}{\pi L f}
$$

3.4. Experimental data and conclusions. As mentioned above, the parallel-plate line is a fast-wave structure with an index of refraction $n_{0}=0.87$. According to section 1.1, a hysteresis should still occur for higher values of the density. However, at such values of the density $\left(\alpha>10^{-3}\right)$ the attenuation in our experiment is large: about $10 \mathrm{~dB}$ over one wavelength. The dependence of the permittivity on $\beta$, sketched as a dotted line in fig. 11, suggests a loss-dominated behaviour ${ }^{11,12}$ ), causing the relativistic effects to be obscured. These measurements are not of further interest to this study.

Our further measurements have been taken for lower values of the density $\left(\alpha \simeq 10^{-4}\right)$. The permittivity of the plasma is close to 1 and the condition $|1-\varepsilon| \ll\left|1-n_{0}^{2}\right|$ is fulfilled, cf. eq. (16). In this regime a hysteresis should not occur but the resonance should be shifted towards higher values of $\beta$. This is, indeed, observed in fig. 11, full line. The values of $\beta_{0}$ where $\varepsilon-1=0$ are shown in fig. 12 as a function of the input power $P_{\text {in }}$. In the range $1 \mathrm{~W}$ to $20 \mathrm{~W}, \beta_{0}$ is proportional to $P_{\text {in }}^{\hbar}$ in agreement with eq. (15). From eq. (15) and fig. 12 an effective value of $\hat{E}$ can be obtained:

$$
\hat{E}=1100 \sqrt{ } P_{\text {in }},
$$

which is 1.9 times the value of $\hat{E}$ derived in eq. (37). The fact that the effective value of $\hat{E}$ is larger than the value of $\hat{E}$ of eq. (37) may be due to the presence of the left-handed part of the wave, to field concentration (radial and axial), and to an increased value of $Z_{0}$ because of the presence of wave guide modes. In the presentation of the results, as described below, we have taken the value of $\hat{E}$ given in eq. (46) for the calculation of $G, c t$. eq. (38).

No measurements have been taken below i $W$ where the shift becomes comparable to that associated with the small inhomogeneity of the static magnetic field. Above the power level of $20 \mathrm{~W}$ the value is independent of $P_{\text {in. }}$. This can be explained by the fact that above this level the radius of gyration of the electrons becomes comparable to half the spacing between the plates of the parallel-plate transmission line. 


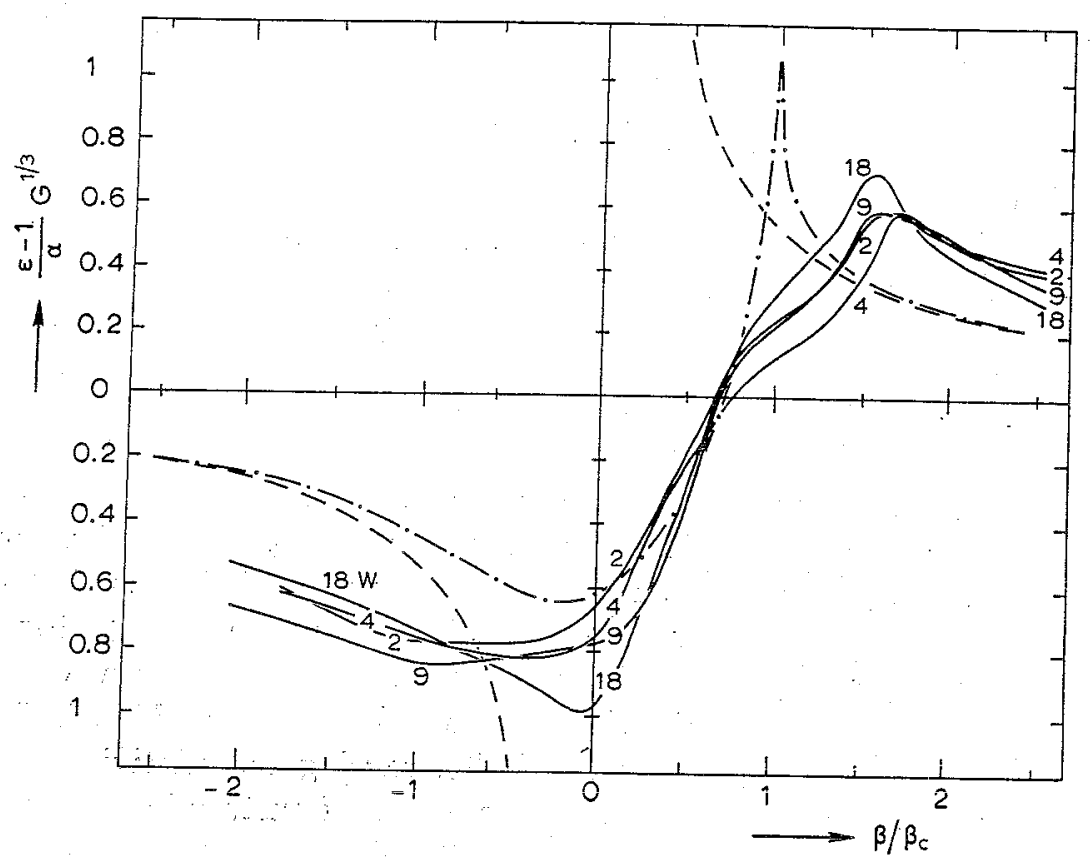

Fig. 13. Measured dependence of $(\varepsilon-1) G^{\frac{x}{3}} / \alpha$ on $\beta / \beta_{\mathrm{c}}$ in the travelling wave experiment for $P_{\text {in }}=2,4,9$, and $18 \mathrm{~W}$. The dash-dot curve represents the theoretical function, $i_{2} / i_{0}$, and the dashed curve is the nonrelativistic equivalent, which follows from $\varepsilon-1=\alpha / \beta$.

Below $20 \mathrm{~W}$ the permittivity, the loss tangent, and the density are measured as functions of $\beta$. The measured quantities are reduced to $(\varepsilon-1) G^{\frac{3}{3}} / \alpha$ and $(\operatorname{tg} \delta / \alpha) G^{\frac{1}{3}}$, and are plotted as functions of $\beta / \beta_{\mathrm{c}}$ in figs. 13 and 14 , respectively. A comparison is made with the theoretical curves $i_{2} / i_{0}$ and $\delta_{\text {extr }}^{\prime} / 2 p i_{0}$, the latter for two fixed values of $p$ and the value of $p$ for axial escape (ct. eqs. (20) and (40)) : $p=3.6 / i_{1}$.

Besides numerical discrepancies, which may be due, e.g., to inaccuracies in the density nieasurements, the predicted dielectric behaviour is well confirmed in the experiment. As predicted theoretically the curves for various values of $P_{1 \text { in }}$ coincide, when plotted in the normalized coordinates. From fig. 14 we may draw the conclusion that here axial escape due to radiation pressure is not the dominant loss mechanism. Possibly, the action of the radiation pressure is impeded by the presence of residual standing waves. The experimental curves suggest a fixed vaiue of $p$ close to 1 . This can be explained by transverse loss of electrons to the plates of the transmission line.

Acknowledgements. I should like to thank Professor C. M. Braams for his stimulating interest. I am very grateful to Dr. L. Th. M. Ornstein 


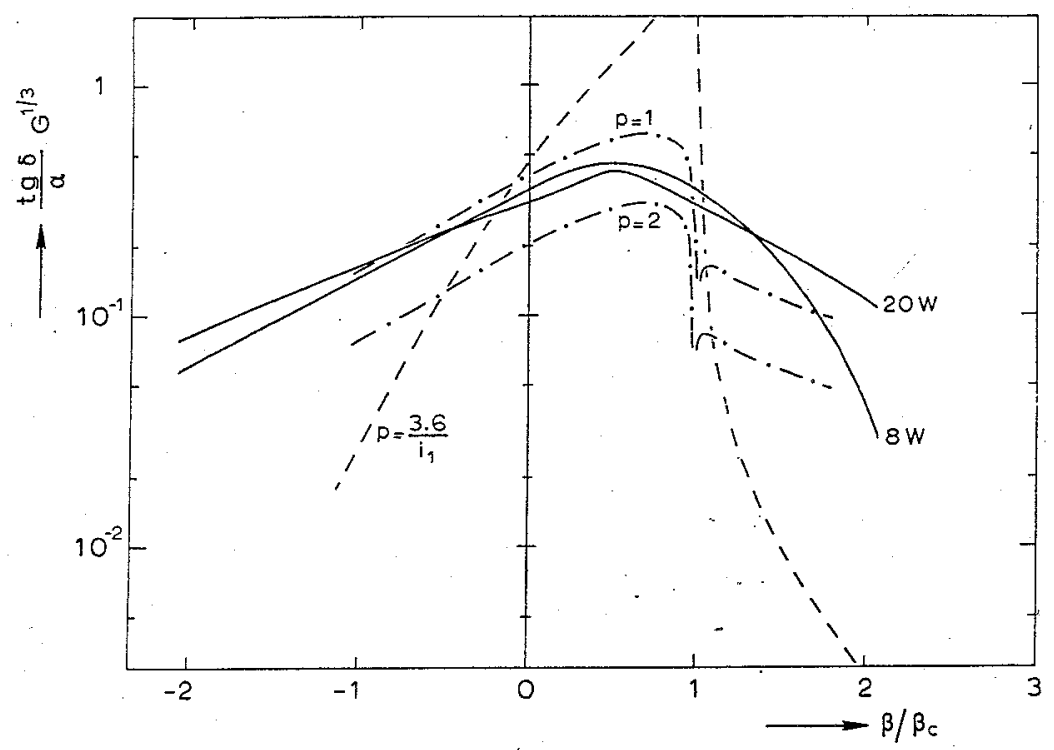

Fig. 14. Measured dependence of $(\operatorname{tg} \delta / \alpha) G^{\frac{1}{3}}$ on $\beta / \beta_{\mathrm{e}}$ in the travelling wave experiment, for $P_{\text {in }}=8$ and $20 \mathrm{~W}$. The dash-dot curves labelled $p=1,2$ give the predicted loss factor for fixed values of $p$. The broken curve labelled $p=3.6 / i_{1}$ shows the loss factor calculated for axial escape (cf. eq. (20)).

for many valuable suggestions and discussions. I am indebted to Mr. P. Manintveld and to Mr. E. Oord for their skillful assistance in building the experiments and in performing the measurements. Thanks are also due to Miss T. J. S. H. Tjin Soe Sjoen, Mr. P. A. van Kuyk and Mr. W. Tukker for the careful preparation of the manuscript.

This work was performed as part of the research programme of the association agreement of Euratom and the "Stichting voor Fundamenteel Onderzoek der Materie" (FOM) with financial support from the "Nederlandse Organisatie voor Zuiver-Wetenschappelijk Onderzoek" (ZWO) and Euratom.

\section{REFERENCES}

1) Ginzburg, V. L., Propagation of e.m. waves in plasma, North-Holland Publishing Co. (Amsteruāra, 19ói).

2) Davydovskii, V. Ya., Soviet Physics-JETP 16 (1963) 629.

3) Roberts, C. S. and Buchsbaum, S. J., Phys. Rev. 135 (1964) A381.

4) Schram, D. C., Physica 40 (1968) 422.

5) Schram, D. C., Rijnhuizen Report 65-25 (1965).

6) Schram, D. C. and Schrader, W. J., 7th Int. Conf. Phen. Ion. Gases - Beograd (1965) Vol. II, 239. 


\section{THE PERMITTIVITY OF A PLASMA AT CYCLOTRON RESONANCE}

7) Schram, D. C. and Beukema, G. P., 2nd Coll. int. sur les interactions entre les champs oscillants et les plasmas, Saclay (1968) Vol. I, 149.

8) Schrader, W. J., Thesis Rijksuniversiteit Utrecht, 1967, Rijnhuizen Report 67-40 (1967).

9) Buchsbaum, S. J., Mower, L. and Brown, S. C., Phys. Fluids 3 (1960) 806.

10) Schrader, W. J., Physica 40 (1968) 223.

11) Wharton, C. B., Chapter 12 of Plasma Physics, Ed. Drummond, J. E., McGraw-Hill (New York, 1961).

12) Bachynski, M. P. and Gibbs, B. W., Plasma Physics (J. nuclear Energy G) 8 (1966) 533. 